\title{
Existence and Multiplicity of Positive Solutions to a Quasilinear Elliptic Equation with Strong Allee Effect Growth Rate
}

\author{
Chan-Gyun Kim and Junping Shi
}

\begin{abstract}
In this paper we consider a $p$-Laplacian equation with strong Allee effect growth rate and Dirichlet boundary condition

$$
\begin{cases}\operatorname{div}\left(|\nabla u|^{p-2} \nabla u\right)+\lambda f(x, u)=0, & x \in \Omega, \\ u=0, & x \in \partial \Omega,\end{cases}
$$

where $\Omega$ is a bounded smooth domain in $\mathbb{R}^{N}$ for $N \geq 1, p>1$, and $\lambda$ is a positive parameter. By using variational methods and a suitable truncation technique, we prove that problem $\left(P_{\lambda}\right)$ has at least two positive solutions for large parameter and it has no positive solutions for small parameter. In addition, a nonexistence result is investigated.
\end{abstract}

Mathematics Subject Classification (2000). 35J92, 35J20, 35J25.

Keywords. p-Laplacain, Allee effect, positive solutions, multiplicity.

\section{Introduction}

Consider a boundary value problem

$$
\begin{cases}\operatorname{div}\left(|\nabla u|^{p-2} \nabla u\right)+\lambda f(x, u)=0, & x \in \Omega, \\ u=0, & x \in \partial \Omega,\end{cases}
$$

where $\Omega$ is a bounded smooth domain in $\mathbb{R}^{N}$ for $N \geq 1, p>1$, and $\lambda$ is a positive parameter.

Partially supported by National Research Foundation of Korea Grant funded by the Korean Government (Ministry of Education, Science and Technology, NRF-2011-357-C00006).

Partially supported by NSF grant DMS-1022648. 
The existence, nonexistence and/or multiplicity of positive solutions to problem $\left(P_{\lambda}\right)$ have been studied extensively in the literature; see, for example, $[1,2,5,6,8,9,11-15,17-25]$ and the references therein. In many previous studies, the nonlinear function $f(x, s) / s^{p-1}$ was assumed to be nonincreasing in $s$, and under this condition, it can be shown that problem $\left(P_{\lambda}\right)$ has at most one positive solution (see, e.g., $[1,6,8,9,22])$. On the other hand, the uniqueness of positive solution no longer holds if $f(x, s) / s^{p-1}$ is not nonincreasing in $s$. In $[12,24,25]$, it was shown that problem $\left(P_{\lambda}\right)$ has at least two positive solutions for sufficiently large $\lambda$ if the nonlinearity $f(x, s)$ is homogeneous, i.e., $f(x, s)=f(s)$, and it satisfies that $f(0)=f(\alpha)=0$ for some $\alpha>0, \lim _{s \rightarrow 0+} f(s) / s^{p-1}=0, f>0$ in $(0, \alpha)$ and $f<0$ in $(\alpha, \infty)$. In [14], it was shown that problem $\left(P_{\lambda}\right)$ has at least two positive solutions for sufficiently large $\lambda$ if $p>2$ and homogeneous nonlinearity $f(x, u)=f(u)$ satisfies that $f(0)=0, \lim _{s \rightarrow 0+} f(s) / s^{p-1}=-m<0$, and there are precisely two numbers $0<\rho_{1}<\rho_{2}$ such that $f\left(\rho_{1}\right)=f\left(\rho_{2}\right)=0, f<0$ in $\left(0, \rho_{1}\right), f>0$ in $\left(\rho_{1}, \rho_{2}\right)$ and $\int_{0}^{\rho_{2}} f(s) d s>0$.

Throughout this paper, we assume that $f(x, u)$ satisfies

(f1) $f \in C(\bar{\Omega} \times[0, \infty), \mathbb{R})$;

(f2) There exist $b(x), c(x) \in C(\bar{\Omega})$ such that $0<b(x)<c(x) \leq M$ for some constant $M>0$, and $f(x, 0)=f(x, b(x))=f(x, c(x))=0$ for all $x \in \bar{\Omega}$;

(f3) For all $x \in \bar{\Omega}, f(x, s)<0$ for any $s \in(0, b(x)) \cup(c(x), \infty)$, and $f(x, s)>0$ for any $s \in(b(x), c(x))$;

(f4) There exists $N_{1}>0$ such that $f(x, s) \geq-N_{1} s^{p-1}$ for all $x \in \bar{\Omega}$ and $0 \leq s \leq M$

(f5) There exists an open ball $B_{1}$ of $\Omega$ such that $c(x) \in C^{1}\left(\bar{B}_{1}\right)$ and

$$
F(x, c(x))>0, \quad x \in B_{1},
$$

where

$$
F(x, s)=\int_{0}^{s} f(x, \tau) d \tau \quad \text { for }(x, s) \in \Omega \times[0, \infty) .
$$

Using variational methods and a suitable truncation technique, we show that problem $\left(P_{\lambda}\right)$ has at least two positive solutions for sufficiently large $\lambda$ and problem $\left(P_{\lambda}\right)$ has no positive solutions for small $\lambda$ when inhomogeneous nonlinearity $f(x, u)$ satisfies $(f 1)-(f 5)$. Our result extends the result of [17] for $p=2$ to $p>1$, and it also extends the result of [14] for $p>2$ and homogeneous nonlinearity to $p>1$ and inhomogeneous nonlinearity.

\section{Preliminaries}

In this section we will establish some basic setups and preliminary results concerning the $p$-Laplacian problems (see, e.g., [7]). Consider a boundary value problem 


$$
\begin{cases}\operatorname{div}\left(|\nabla u|^{p-2} \nabla u\right)+g(x, u)=0, & x \in \Omega, \\ u=0, & x \in \partial \Omega,\end{cases}
$$

where $p>1$ and suppose $g: \Omega \times \mathbb{R} \rightarrow \mathbb{R}$ is a Carathéodory function and it satisfies the growth condition:

$$
|g(x, s)| \leq C|s|^{q-1}+b(x) \quad \text { for }(x, s) \in \Omega \times \mathbb{R},
$$

where $C \geq 0$ is constant, $q>1, b \in L^{q^{\prime}}(\Omega), 1 / q+1 / q^{\prime}=1$.

A function $u \in W_{0}^{1, p}(\Omega)$ is said to be a solution of problem $(Q)$ if

$$
\int_{\Omega}|\nabla u|^{p-2} \nabla u \nabla v d x=\int_{\Omega} g(x, u) v d x \quad \text { for all } v \in W_{0}^{1, p}(\Omega) .
$$

Define $\Phi: W_{0}^{1, p}(\Omega) \rightarrow \mathbb{R}$ by

$$
\Phi(u)=\int_{\Omega} G(x, u(x)) d x \quad \text { for } u \in W_{0}^{1, p}(\Omega),
$$

where $G: \Omega \times \mathbb{R} \rightarrow \mathbb{R}$ is defined by

$$
G(x, s)=\int_{0}^{s} g(x, \tau) d \tau \quad \text { for }(x, s) \in \Omega \times \mathbb{R} .
$$

Then $\Phi$ is continuously Fréchet differentiable on $W_{0}^{1, p}(\Omega)$ if $g(x, s)$ satisfies (2.1) with $q \in\left(1, p^{*}\right)$, where

$$
p^{*}= \begin{cases}\frac{N p}{N-p}, & p<N \\ \infty, & p \geq N\end{cases}
$$

and

$$
\Phi^{\prime}(u) \phi=\int_{\Omega} g(x, u) \phi d x \quad \text { for } u, \phi \in W_{0}^{1, p}(\Omega) .
$$

Consequently, the functional $I: W_{0}^{1, p}(\Omega) \rightarrow \mathbb{R}$ defined by

$$
I(u)=\frac{1}{p} \int_{\Omega}|\nabla u|^{p} d x-\Phi(u) \quad \text { for } u \in W_{0}^{1, p}(\Omega)
$$

is continuously Fréchet differentiable on $W_{0}^{1, p}(\Omega)$ if $g(x, s)$ satisfies (2.1) with $q \in\left(1, p^{*}\right)$, and

$$
I^{\prime}(u) \phi=\int_{\Omega}|\nabla u|^{p-2} \nabla u \nabla \phi d x-\int_{\Omega} g(x, u) \phi d x \quad \text { for all } \phi \in W_{0}^{1, p}(\Omega) .
$$

Thus critical points of $I$ are solutions of $(Q)$.

We define $u \in W^{1, p}(\Omega)$ to be a sub-solution to problem $(Q)$ if $u \leq 0$ on $\partial \Omega$ and

$$
\int_{\Omega}|\nabla u|^{p-2} \nabla u \nabla \phi d x-\int_{\Omega} g(x, u) \phi d x \leq 0 \quad \text { for all } \phi \in W_{0}^{1, p}(\Omega), \phi \geq 0 .
$$

Similarly, $u \in W^{1, p}(\Omega)$ is a super-solution to problem $(Q)$ if in the above the reverse inequalities hold. 
Finally we recall the following existence result based on super-subsolution method: ([10, Theorem 4.11]).

Theorem 2.1. Assume that $g(x, s)$ satisfies (2.1) with $q=p$, and assume that $\rho \in W^{1, p}(\Omega) \cap L^{\infty}(\Omega)$ is a sub-solution and $\psi \in W^{1, p}(\Omega) \cap L^{\infty}(\Omega)$ is a supersolution to $(Q)$ such that $\rho \leq \psi$. Then $(Q)$ has a minimal solution $u_{*}$ and $a$ maximal solution $u^{*}$ in the order interval $[\rho, \psi]$ such that any solution $u$ of $(Q)$ in $[\rho, \psi]$ satisfies $u_{*} \leq u \leq u^{*}$.

\section{Main Results}

Consider the following truncation of the nonlinearity of $f(x, s)$ :

$$
\hat{f}(x, s):= \begin{cases}0, & (x, s) \in \bar{\Omega} \times(-\infty, 0], \\ f(x, s), & (x, u) \in \bar{\Omega} \times(0, M], \\ f(x, M), & (x, s) \in \bar{\Omega} \times(M, \infty) .\end{cases}
$$

Then for each $q \in\left(1, p^{*}\right)$, there exists a constant $C(q)>0$ such that

$$
\hat{f}(x, s) \leq C(q)|s|^{q-1}, \quad(x, s) \in \Omega \times \mathbb{R} .
$$

Define the functional $\hat{I}_{\lambda}: W_{0}^{1, p}(\Omega) \rightarrow \mathbb{R}$ by

$$
\hat{I}_{\lambda}(u):=\frac{1}{p} \int_{\Omega}|\nabla u(x)|^{p} d x-\lambda \int_{\Omega} \hat{F}(x, u(x)) d x, \quad u \in W_{0}^{1, p}(\Omega),
$$

where $\hat{F}(x, s)=\int_{0}^{s} \hat{f}(x, \tau) d \tau,(x, s) \in \Omega \times \mathbb{R}$. Since $\hat{f}(x, s)$ is bounded, the functional $\hat{I}_{\lambda}$ is continuously Fréchet differentiable on $W_{0}^{1, p}(\Omega)$, and it is also weakly lower-semicontinuous and coercive on $W_{0}^{1, p}(\Omega)$. Moreover, the functional $\hat{I}_{\lambda}$ satisfies the Palais-Smale condition. Indeed, let $\left\{u_{n}\right\}$ be any sequence in $W_{0}^{1, p}(\Omega)$ such that $\left\{\hat{I}_{\lambda}\left(u_{n}\right)\right\}$ is bounded and $\hat{I}_{\lambda}^{\prime}\left(u_{n}\right) \rightarrow 0$ as $n \rightarrow \infty$. Then it follows from the boundedness of $\hat{F}$ that $\left\{u_{n}\right\}$ is bounded in $W_{0}^{1, p}(\Omega)$. By Lemma 2 on page 363 of [7], the sequence $\left\{u_{n}\right\}$ has a convergent subsequence, and thus the functional $\hat{I}_{\lambda}$ satisfies the Palais-Smale condition.

Let $u$ be any critical point of $\hat{I}_{\lambda}$. Then $0 \leq u(x) \leq M$ by the same argument as in the proof of [25, Propsition 2.1]. Here we use the facts that $\hat{f}(x, s)=$ 0 for all $(x, s) \in \Omega \times(-\infty, 0]$ and $\hat{f}(x, s) \leq 0$ for all $(x, s) \in \Omega \times[M, \infty)$, and $M$ is the constant in the condition $(f 2)$. Thus $u$ is a nonnegative bounded solution of $\left(P_{\lambda}\right)$, and $u \in C_{0}^{1, \beta}(\bar{\Omega})$ for some $\beta \in(0,1)$ by Lieberman's regularity result [16, Theorem 1]. It follows from the maximum principle due to Vázquez [26, Theorem 5] that $u>0$ in $\Omega$ and $\partial u / \partial \nu<0$ on $\partial \Omega$ if $u \not \equiv 0$ in $\Omega$.

Theorem 3.1. Let $p>1$ and suppose that $f(x, s)$ satisfies $(f 1)-f(5)$. Then problem $\left(P_{\lambda}\right)$ has at least two positive solutions for sufficiently large $\lambda$, and it 
has no positive solutions for $\lambda \leq \lambda_{1} / C(p)$. Here $C(p)$ is the constant in (3.1) with $q=p$, and $\lambda_{1}>0$ is the principal eigenvalue of the problem

$$
\begin{cases}\operatorname{div}\left(|\nabla u|^{p-2} \nabla u\right)+\lambda|u|^{p-2} u=0, & x \in \Omega, \\ u=0, & x \in \partial \Omega\end{cases}
$$

with the associated eigenfunction $\phi_{1}>0$ in $\Omega$.

Proof. Since $\hat{I}_{\lambda}$ is weakly lower-semicontinuous and coercive on $W_{0}^{1, p}(\Omega), \hat{I}_{\lambda}$ has a global minimizer $u_{1} \in W_{0}^{1, p}(\Omega)$. We will show that for large $\lambda$, there exists $v \in W_{0}^{1, p}(\Omega)$ such that $\hat{I}_{\lambda}(v)<0=\hat{I}_{\lambda}(0)$. Then $u_{1} \not \equiv 0$, and thus $u_{1}$ is a positive solution of problem $\left(P_{\lambda}\right)$ for large $\lambda$.

For small $\epsilon>0$, we define

$$
v_{\epsilon}(x):= \begin{cases}0, & x \in \Omega \backslash B_{1}^{\epsilon} \\ c_{\epsilon}(x), & x \in B_{1}^{\epsilon} \backslash \bar{B}_{1} \\ c(x), & x \in B_{1},\end{cases}
$$

where $B_{1}^{\epsilon}:=\left\{x \in \Omega: \operatorname{dist}\left(x, B_{1}\right) \leq \epsilon\right\}, B_{1}$ is the open set in $(f 4), c(x)$ is the function in $(f 2)$ and $c_{\epsilon}(x)$ is an appropriate function such that $0 \leq v_{\epsilon}(x) \leq$ $c(x), x \in \Omega$ and $v_{\epsilon} \in C_{0}^{1}(\bar{\Omega})$. Then $\hat{F}\left(x, v_{\epsilon}(x)\right)=F\left(x, v_{\epsilon}(x)\right), x \in \Omega$ and

$$
\begin{aligned}
\hat{I}_{\lambda}\left(v_{\epsilon}\right) & =\frac{1}{p} \int_{\Omega}\left|\nabla v_{\epsilon}(x)\right|^{p} d x-\lambda \int_{\Omega} F\left(x, v_{\epsilon}(x)\right) d x \\
& =\frac{1}{p} \int_{\Omega}\left|\nabla v_{\epsilon}(x)\right|^{p} d x-\lambda \int_{B_{1}} F(x, c(x)) d x-\lambda \int_{B_{1}^{\epsilon} \backslash \bar{B}_{1}} F\left(x, c_{\epsilon}(x)\right) d x \\
& \leq \frac{1}{p} \int_{\Omega}\left|\nabla v_{\epsilon}(x)\right|^{p} d x-\lambda \int_{B_{1}} F(x, c(x)) d x+\lambda A\left|B_{1}^{\epsilon} \backslash \bar{B}_{1}\right|
\end{aligned}
$$

where $A=\max _{0 \leq u \leq M}|F(x, u)|$. Since $F(x, c(x))>0$ is continuous in $B_{1}$, there exist an open subset $B_{0}$ with $\bar{B}_{0} \subseteq B_{1}$ and a constant $\delta_{0}>0$ such that $\left|B_{0}\right|>0$ and $F(x, c(x)) \geq \delta_{0}$ for $x \in B_{0}$. Choose a sufficiently small $\epsilon_{0}>0$ so that

$$
\delta_{0}\left|B_{0}\right|-A\left|B_{1}^{\epsilon_{0}} \backslash \bar{B}_{1}\right|>\frac{\delta_{0}\left|B_{0}\right|}{2}
$$

Then

$$
\begin{aligned}
\hat{I}_{\lambda}\left(v_{\epsilon_{0}}\right) & \leq \frac{1}{p} \int_{\Omega}\left|\nabla v_{\epsilon_{0}}(x)\right|^{p} d x-\lambda \int_{B_{0}} F(x, c(x)) d x+\lambda A\left|B_{1}^{\epsilon_{0}} \backslash \bar{B}_{1}\right| \\
& \leq \frac{1}{p} \int_{\Omega}\left|\nabla v_{\epsilon_{0}}(x)\right|^{p} d x-\lambda\left(\delta\left|B_{0}\right|-A\left|B_{1}^{\epsilon_{0}} \backslash \bar{B}_{1}\right|\right) \\
& \leq \frac{1}{p} \int_{\Omega}\left|\nabla v_{\epsilon_{0}}(x)\right|^{p} d x-\lambda \frac{\delta_{0}\left|B_{0}\right|}{2}
\end{aligned}
$$

which implies that $\hat{I}_{\lambda}\left(v_{\epsilon_{0}}\right)<0$ for sufficiently large $\lambda$. Consequently $\left(P_{\lambda}\right)$ has a positive solution $u_{1}$ satisfying $\hat{I}_{\lambda}\left(u_{1}\right)=\inf \hat{I}_{\lambda}(u)<0$ for all large $\lambda$. 
Fix $q^{*} \in\left(p, p^{*}\right)$. Then it follows from (3.1) that

$$
\hat{F}(x, s) \leq \frac{C\left(q^{*}\right)}{q^{*}}|s|^{q^{*}}, \quad(x, s) \in \Omega \times \mathbb{R},
$$

and for $u \in W_{0}^{1, p}(\Omega)$, by the Sobolev inequality,

$$
\begin{aligned}
\hat{I}_{\lambda}(u) & \geq \frac{1}{p}\|u\|_{W_{0}^{1, p}}^{p}-\frac{\lambda C\left(q^{*}\right)}{q^{*}} \int_{\Omega}|u(x)|^{q^{*}} d x \\
& \geq \frac{1}{p}\left[1-\lambda C_{1}\|u\|_{W_{0}^{1, p}}^{q^{*}-p}\right]\|u\|_{W_{0}^{1, p}}^{p}
\end{aligned}
$$

for some constant $C_{1}>0$. Thus for each $\lambda>0$, there exists $\rho>0$ such that $\hat{I}_{\lambda}(u)>0=\hat{I}_{\lambda}(0)$ if $0<\|u\|_{W_{0}^{1, p}} \leq \rho$. Fix $\lambda>0$ such that $\hat{I}_{\lambda}\left(u_{1}\right)<0$. It follows from Mountain pass lemma that $\hat{I}_{\lambda}$ has another critical point $u_{2}$ such that

$$
\hat{I}_{\lambda}\left(u_{2}\right)>0>\hat{I}_{\lambda}\left(u_{1}\right)
$$

and thus problem $\left(P_{\lambda}\right)$ has another positive solution $u_{2}$ for all large $\lambda$.

Finally we show that problem $\left(P_{\lambda}\right)$ has no positive solution for all $\lambda \leq$ $\lambda_{1} / C(p)$. Assume on the contrary that there exists a positive solution $u_{\lambda}$ of problem $\left(P_{\lambda}\right)$ such that $\lambda \leq \lambda_{1} / C(p)$. Let $u=u_{\lambda}, v=\phi_{1}, A=B=1, a(x)=$ $\lambda f\left(x, u_{\lambda}(x)\right) / u_{\lambda}(x)^{p-1}, b(x)=\lambda_{1}$ in $[3$, Theorem 1]. Then $a(x) \leq b(x)$ in $\Omega$, and

$$
\int_{\Omega} L\left(u_{\lambda}, \phi_{1}\right) d x \leq 0
$$

since $\phi_{1}>0$ in $\Omega$. Here

$$
L\left(u_{\lambda}, \phi_{1}\right):=\left|\nabla u_{\lambda}\right|^{p}-p\left(\frac{u_{\lambda}}{\phi_{1}}\right)^{p-1}\left|\phi_{1}\right|^{p-2} \nabla \phi_{1} \nabla u_{\lambda}+(p-1)\left(\frac{u_{\lambda}}{\phi_{1}}\right)^{p}\left|\nabla \phi_{1}\right|^{p} .
$$

On the other hand, $L\left(u_{\lambda}, \phi_{1}\right) \geq 0$ by Picone's identity (see, e.g., [4, Theorem 1.1]). Thus $L\left(u_{\lambda}, \phi_{1}\right)=0$, for a.e. in $\Omega$, which implies $u_{\lambda}=k \phi_{1}$ for some constant $k$, and one can easily proceed a contradiction.

As an example of Theorem 3.1, we consider the following inhomogeneous cubic nonlinearity case:

$$
\begin{cases}\operatorname{div}\left(|\nabla u|^{p-2} \nabla u\right)+\lambda u^{p-1}(u-b(x))(c(x)-u)=0, & x \in \Omega, \\ u=0, & x \in \partial \Omega\end{cases}
$$

where $b(x), c(x) \in C(\bar{\Omega})$ such that $0<b(x)<c(x)$ for any $x \in \bar{\Omega}$. 
It is easy to verify that $f(x, s)=s^{p-1}(s-b(x))(c(x)-s)$ satisfies $(f 1)-$ $(f 4)$. Moreover $f$ satisfies $(f 5)$ if there exists an open ball $B_{1} \subseteq \Omega$ such that $c(x) \in C^{1}\left(\bar{B}_{1}\right)$ and

$$
0<\left(1+\frac{2}{p}\right) b(x)<c(x) \text { in } B_{1} .
$$

Then by Theorem 3.1, problem (3.2) has at least two positive solutions for large $\lambda$, and no positive solutions for small $\lambda$.

Finally we give the nonexistence of the positive solutions of problem $\left(P_{\lambda}\right)$ when $(f 5)$ does not hold. We define $\bar{f}(s):=\max _{x \in \bar{\Omega}} f(x, s), b_{*}:=\min _{x \in \bar{\Omega}} b(x)$ and $c^{*}:=\max _{x \in \bar{\Omega}} c(x)$. Then $\bar{f}$ is a continuous function on $[0, \infty)$, and it satisfies that $\bar{f}(0)=\bar{f}\left(b_{*}\right)=\bar{f}\left(c^{*}\right)=0, \bar{f}(s)<0$ for any $s \in\left(0, b_{*}\right) \cup\left(c^{*}, \infty\right)$, and $\bar{f}(s)>0$ for any $s \in\left(b_{*}, c^{*}\right)$ when $f$ satisfies $(f 1)-(f 3)$.

Theorem 3.2. Let $p>1$ and suppose that $f(x, s)$ satisfies $(f 1)-f(3)$. If $\int_{0}^{c^{*}} \bar{f}(s) d s<0$, then problem $\left(P_{\lambda}\right)$ has no positive solutions for any $\lambda>0$.

Proof. Assume on the contrary that there exists a positive solution $\left(\lambda, u_{\lambda}\right)$ of problem $\left(P_{\lambda}\right)$. Then $u_{\lambda}$ is a sub-solution of

$$
\begin{cases}\operatorname{div}\left(|\nabla u|^{p-2} \nabla u\right)+\lambda \bar{f}(u)=0, & x \in \Omega, \\ u=0, & x \in \partial \Omega,\end{cases}
$$

and $c^{*}$ is a super-solution of problem (3.3). Since $\left\|u_{\lambda}\right\|_{\infty} \in\left(b_{*}, c^{*}\right]$, problem (3.3) has a positive solution $u^{*}$ such that $u_{\lambda} \leq u^{*} \leq c^{*}$ in view of Theorem 2.1. On the other hand, it follows from Loc and Schmitt [18, Remark 2] that $\int_{0}^{c^{*}} \bar{f}(s) d s \geq 0$, which contradicts the hypothesis $\int_{0}^{c^{*}} \bar{f}(s) d s<0$.

\section{References}

[1] Afrouzi, G.A., Rasouli, S.H.: A remark on the uniqueness of positive solutions for some Dirichlet problems. Nonlinear Anal. 64(12), 2773-2777 (2006)

[2] Ali, J., Shivaji, R., Wampler, K.: Population models with diffusion, strong Allee effect and constant yield harvesting. J. Math. Anal. Appl. 352(2), 907-913 (2009)

[3] Allegretto, W.: Sturm theorems for degenerate elliptic equations. Proc. Amer. Math. Soc. 129(10):3031-3035 (electronic) (2001)

[4] Allegretto, W., Huang, Y.X.: A Picone's identity for the $p$-Laplacian and applications. Nonlinear Anal. 32(7), 819-830 (1998)

[5] Dancer, E.N., Schmitt, K.: On positive solutions of semilinear elliptic equations. Proc. Amer. Math. Soc. 101(3), 445-452 (1987)

[6] Díaz, J.I., Saá, J.E.: Existence et unicité de solutions positives pour certaines équations elliptiques quasilinéaires. C. R. Acad. Sci. Paris Sér. I Math. 305(12), 521-524 (1987)

[7] Dinca, G., Jebelean, P., Mawhin, J.: Variational and topological methods for Dirichlet problems with $p$-Laplacian. Port. Math. (N.S.) 58(3), 339-378 (2001) 
[8] Drábek, P., Hernández, J.: Existence and uniqueness of positive solutions for some quasilinear elliptic problems. Nonlinear Anal. 44(2), 189-204 (2001)

[9] Drábek, P., Krejčí, P., Takáč, P.: Nonlinear differential equations, volume 404 of Chapman \& Hall/CRC research notes in mathematics. Chapman \& Hall/CRC, Boca Raton, FL, 1999. Papers from the seminar on differential equations held in Chvalatice, June 29-July 3 (1998)

[10] Du, Y.: Order structure and topological methods in nonlinear partial differential equations. Maximum principles and applications. Series in partial differential equations and applications. vol. 1. World Scientific Publishing Co. Pte. Ltd., Hackensack, NJ (2006)

[11] Goddard II, J., Lee, E.K., Shivaji, R.: Population models with diffusion, strong Allee effect, and nonlinear boundary conditions. Nonlinear Anal. 74(17), 62026208 (2011)

[12] Guo, Z.: Some existence and multiplicity results for a class of quasilinear elliptic eigenvalue problems. Nonlinear Anal. 18(10), 957-971 (1992)

[13] Guo, Z.: Exact multiplicity for some quasilinear elliptic Dirichlet problems where the non-linearity changes sign. Nonlinear Anal. 61(7), 1135-1155 (2005)

[14] Guo, Z., Webb, J.R.L.: Large and small solutions of a class of quasilinear elliptic eigenvalue problems. J. Differ. Equ. 180(1), 1-50 (2002)

[15] Hung, K.C., Wang, S.H.: Bifurcation diagrams of a $p$-Laplacian Dirichlet problem with Allee effect and an application to a diffusive logistic equation with predation. J. Math. Anal. Appl. 375(1), 294-309 (2011)

[16] Lieberman, G.M.: Boundary regularity for solutions of degenerate elliptic equations. Nonlinear Anal. 12(11), 1203-1219 (1988)

[17] Liu, G., Wang, Y., Shi, J.: Existence and nonexistence of positive solutions of semilinear elliptic equation with inhomogeneous strong Allee effect. Appl. Math. Mech. (English Ed.) 30(11), 1461-1468 (2009)

[18] Loc, N.H., Schmitt, K.: On positive solutions of quasilinear elliptic equations. Differ. Integral Equ. 22(9-10), 829-842 (2009)

[19] Oruganti, S., Shi, J., Shivaji, R.: Logistic equation wtih the $p$-Laplacian and constant yield harvesting. Abstr. Appl. Anal. (9):723-727 (2004)

[20] Ouyang, T., Shi, J.: Exact multiplicity of positive solutions for a class of semilinear problems. J. Differ. Equ. 146(1), 121-156 (1998)

[21] Ouyang, T., Shi, J.: Exact multiplicity of positive solutions for a class of semilinear problem. II.. J. Differ. Equ. 158(1), 94-151 (1999)

[22] Rynne, B.P.: A global curve of stable, positive solutions for a $p$-Laplacian problem. Electron. J. Differ. Equ. 58, 1-12 (2010)

[23] Shi, J., Shivaji, R.: Persistence in reaction diffusion models with weak Allee effect. J. Math. Biol. 52(6), 807-829 (2006)

[24] Takeuchi, S.: Multiplicity result for a degenerate elliptic equation with logistic reaction. J. Differ. Equ. 173(1), 138-144 (2001) 
[25] Takeuchi, S.: Positive solutions of a degenerate elliptic equation with logistic reaction. Proc. Amer. Math. Soc. 129(2):433-441 (electronic) (2001)

[26] Vázquez, J.L.: A strong maximum principle for some quasilinear elliptic equations. Appl. Math. Optim. 12(3), 191-202 (1984)

Chan-Gyun Kim and Junping Shi

Department of Mathematics

College of William and Mary

Williamsburg, VI 23187-8795

USA

e-mail: cgkim75@gmail.com;

shij@math.wm.edu

Received: September 7, 2012.

Accepted: February 2, 2013. 